\title{
A Survey of Manufacturing and Handling Practices for Monoclonal Antibodies (MABs) by pharmacy, nursing and medical personnel
}

M Alexander, J King, S Lingaratnam, J Byrne, K MacMillan, A Mollo, S Kirsa, M Green

Marliese Alexander, B.Pharm, Grad Cert Pharm Prac, MPH, Pharmacy Department Peter MacCallum Cancer Centre, Melbourne, Australia

Julie King, B. Pharm, Pharmacy Department, Western Health, Melbourne, Australia

Senthil Lingaratnam, B.Pharm, MPH, Pharmacy Department Peter MacCallum Cancer Centre, Melbourne, Australia

Jenny Byrne, Western \& Central Melbourne Integrated Cancer Service, Melbourne, Australia

Kent MacMillan, B. Pharm, M.Sc, Pharmacy Department, Western Health, Melbourne, Australia

Adele Mollo, Cancer, Neurology/Stroke, Respiratory \& Sub Specialty Medicine, Western Health, Melbourne, Australia

Sue Kirsa, B.Pharm, Grad Dip Hosp Pharm, FSHP, Pharmacy Department Peter MacCallum Cancer Centre, Melbourne, Australia

Michael Green, MBBS FRACP FACP, Department of Cancer Services, Western Health, Melbourne, Australia

Corresponding Author:

Conflicts of Interest:

Funding:

Keywords:

Abstract words:

Words:

Tables:

Figures:

References:
Marliese Alexander

Pharmacy Department, Peter MacCallum Cancer Centre,

Melbourne, Australia

Marliese.Alexander@petermac.org

p 6139656 3583, f 61396561405

Locked Bag 1 A'Beckett Street, Victoria 8006 Australia

The following listed authors areconsultants or advisory committee members or receive honoraria, fees for service, or travel assistance (independent ofresearch-related meetings) from; or have research or other associationswith the organizations listed: Michael Green Roche, Sandoz, Sue Kirsa - Roche, Sandoz, Amgen, Orion, Perigo, Novartis, Senthil Lingaratnam - Roche, Sanofi-Aventis.

This project was funded by Western and Central Melbourne Integrated Cancer Service (WCMICS) as part of a project commissioned to develop safe handling guidelines for monoclonal antibodies.

Cancer, occupational exposure, guidelines, survey, monoclonal antibodies

228

3223

2

4

37 


\section{A survey of manufacturing and handling practices for monoclonal antibodies (MABs) by pharmacy, nursing and medical personnel}

\section{Abstract}

Introduction: There is a paucity of data available to assess the occupational health and safety risk associated with exposure to Monoclonal Antibodies (MABs). Industry standards and published guidelines are conflicting or out-dated. Guidelines offer contrary recommendations based on an array of methodological approaches. This survey aimed to describe current practices, beliefs and attitudes relating to the handling of MABs by Australian medical, nursing and pharmacy clinicians.

Methods: An electronic survey was distributed between June and September 2013. Respondents were surveyed on three focus areas; demographics, institutional guidelines and current practices \& attitudes.

Results: 222 clinicians completed the survey with representation from all targeted professional groups and from a variety of geographic locations. $92 \%$ per cent of respondents reported that their institution prepared or administered MABs, with $87 \%$ specifically handling anti-cancer MABs. MABs were mostly prepared on-site (84\% to $90 \%$ ). $43 \%$ of respondents reported access to institutional MAB handling guidelines with risk reduction strategies including training and education (71\%), spill and waste management (71\%), procedures for transportation $(57 \%)$ and restricted handling $(50 \%)$. Nurses had a stronger preference toward pharmacy manufacturing than both doctors and pharmacists for a range of clinical scenarios. 95\% of all respondents identified that professional or regulatory body guidelines are an important resource when considering handling practices.

Conclusion: MABs are most commonly handled according to cytotoxic drug standards, and often in the absence of formal guidelines. 


\section{Introduction}

Monoclonal antibodies (MABs) are being rapidly introduced into medical oncology and haematology clinical practice with widespread application in the treatment and supportive care of cancer patients. There is a paucity of data available to assess the occupational health and safety risk associated with exposure to these molecules. Dissimilarities in both chemical and physical properties of MABs, as compared with traditional cytotoxic anticancer agents, limit the ability to extrapolate safety data regarding occupational exposure risk. This is particularly relevant when assessing the ability of healthcare workers to internalise (the rate-limiting step in systemic bioavailability and toxicity) these agents in the context of occupational exposure. MABs either do not fulfil conventional hazardous substance criteria or lack sufficient agent-specific information to assign an appropriate hazard classification. Hazardous substance lists and criteria from the National Occupational Health and Safety Commission (NOHSC) of Australia (now Safe Work Australia) and the National Institute for Occupational Safety and Health (NIOSH) of America, do not include any major anticancer MABs. ${ }^{1,2}$ NOHSC criteria were derived from European Community's (EC) legislation for classifying dangerous substances, ${ }^{3-5}$ whilst the NIOSH criteria were adapted from criteria developed by the American Society of Hospital Pharmacists (ASHP). ${ }^{6}$ Withstanding minor differences in terminology and phrasing, both criteria are fundamentally equivocal and consider toxicity (acute and chronic), carcinogenicity, mutagenicity/genotoxicity, and teratogenicity (toxicity to reproduction, fertility and/or development). ${ }^{7,8}$

Australian industry standards for the safe handling and exposure risk associated with MABs are conflicting and out-dated. Occupational health and safety \& dangerous substances legislative documents do not include reference to MABs or other new anticancer agents. ${ }^{7,9-11}$ Similarly, occupational health and safety authorities, under the umbrella of Safe Work Australia, do not provide recommendations relating to the handling of these agents. ${ }^{12}$ The most recent publication of relevance by Work Safe Victoria 'Handling Cytotoxics in the Workplace 2003' does not mention MABs. ${ }^{12}$ A more recent publication by the South Australian government 'Safe Handling of Hazardous Drugs and Related Wastes 2012' discusses MABs however does not provide formal handling recommendations. ${ }^{13}$ The Society of Hospital Pharmacists of Australia (SHPA), recommends that until definitive research proves otherwise, MABs should be handled in dedicated negative pressure (containment) clean areas to prevent exposure. ${ }^{14}$ Material Safety Data Sheets (MSDS) from pharmaceutical companies provide varying levels of recommendations; no special control measures (ofatumumab), ${ }^{15}$ restriction of handling to specific areas and by restricted personnel (cetuximab), ${ }^{16}$ use of personal protective equipment with respiratory protection (ipilimumab) ${ }^{14}$ or without respiratory protection (bevacizumab), ${ }^{17}$ and handling in enclosed processes or within a chemical hood (panitumumab). ${ }^{18}$

In recent years several guidelines (published and unpublished) for the safe handling of MABs have been developed; ${ }^{19-25}$ unfortunately confounding rather than clarifying the issue of safe handling and occupational exposure risks. Guidelines have employed differing methodological approaches considering a range of drug and occupational factors. Handling recommendations have been formulated based on unknown occupational safety risk (i.e. maximum precaution until further evidence) $;^{14} \mathrm{MAB}$ origin; ${ }^{23} \mathrm{MAB}$ origin, toxicity and 
complexity of dosing and manufacturing; ${ }^{25}$ complexity of manufacturing, clinical risk (patient safety) and toxicity; ${ }^{22}$ clinical risk and toxicity; ${ }^{24}$ toxicity and risk of internalisation. ${ }^{20,}{ }^{21}$ Recommendations using these methodologies range from minimal personal protective equipment (gloves) to full cytotoxic precautions.

Consequential to this wide array of recommendations and omissions within key regulatory, industry and professional body documents, correct handling procedures and safety precautions are uncertain. MABs are being handled both according to cytotoxic standards (i.e. pharmacy clean room) and with limited safety precautions (i.e. at the bedside). The default of handling MABs as cytotoxic agents results in the use of handling standards which may be overcautious. Implications include decreased efficiency, increased costs (despite compounding rebates) and time allocation in relation to resources required for preparation (pharmacy personnel time, consumables required, use of cytotoxic drug safety cabinets) and administration (appropriately trained nursing staff, hospital only treatment). Conversely an unconsidered approach may place staff at risk or result in adverse clinical effects and significant financial cost as a result of erroneous manufacturing technique and potential compromise of product integrity. A French survey of handling practices reported that $92 \%$ of MABs were prepared as for cytotoxic agents (hospital pharmacy-based centralised areas, isolators, safety cabinets and exhaust fans). ${ }^{26}$ Interestingly, the same survey found that half of the healthcare personnel regarded their own occupational risk as low.

This study describes current practices, beliefs and attitudes relating to the handling of MABs from the perspective of medical, nursing and pharmacy clinicians in the Australian healthcare setting. The results of this survey were used to guide the development of Australian consensus guidelines for the safe handling and administration of MABs for cancer treatment by healthcare personnel.

\section{Methods:}

Population

Medical, pharmacy and nursing personnel working in Australian healthcare and pharmaceutical manufacturing industries holding current membership with targeted professional organisations; Cancer Nurses Society of Australia (CNSA), Clinical Oncology Society of Australia (COSA), Haematology Society of Australia and New Zealand (HSANZ), Medical Oncology Group of Australia (MOGA) and Society of Hospital Pharmacists of Australia (SHPA).

\section{Study Design}

A multidisciplinary survey of current practice and opinion relating to the handling of MABs was developed using Survey Monkey ${ }^{\circledR}$ software. Peter MacCallum Cancer Centre ethics approval was obtained (approval number: 13/49L). Respondents were surveyed on three focus areas; demographics, institutional guidelines and current practices and attitudes. Demographics included profession (nurse, pharmacist or doctor), specialisation (medical oncology or haematology) and description of primary place of practice (location, number of beds). Availability of institutional guidelines included dichotomous (yes/no) and qualitative assessment. Opinions and 
current practices were established by asking respondents about the handling of a range of MABs (bevacizumab, brentuximab vedotin, cetuximab, denosumab, rituximab, and trastuzumab), chosen to represent the range of available dose forms and manufacturing and administration techniques of commonly used MABs. The survey was piloted locally with assessments providing an opportunity to improve clarity and focus of questions prior to wider distribution.

\section{Data Collection and Analysis}

Distribution of the survey was staggered between June and September 2013 based on timing of approval and distribution of the survey by the relevant professional organisation. In all cases, the survey was distributed electronically with a reminder email after 2 weeks and response cut-off after 8 weeks. The total number of individuals surveyed (and hence response rate) is not reported due to an assumed substantial proportion of co- or multi-membership holders, who would have received the survey multiple times. All responses were anonymous and consent to participate was obtained as the first survey question with automatic termination if consent was not provided. All responses were included in analyses with data aggregated and presented using simple descriptive statistics. Means and $95 \% \mathrm{Cl}$ were used to estimate proportions across the Australian population. Two-sample tests of proportions were used to report on the significanceof differences in responses according to respondentprofession (nursing, pharmacy and medical).

\section{Results:}

Consent and subsequent survey completion was achieved by 222/223 (99.6\%) of clinicians who accessed the survey. Respondents were from across Australia and New Zealand and represented a variety of health services including metropolitan, regional, public and private centres as detailed in table 1. Pharmacy personnel constituted the majority of respondents $(n=113,51 \%)$, followed by nurses $(n=50,22 \%)$ and doctors $(n=46$, 21\%); $13(5 \%)$ respondents did not provide an answer to this question. Detailed respondent professional information is presented in table 1.

The majority of respondents $(205 / 222,92 \%)$ reported that their institution handled (prepared or administered) MABs, with most $(194 / 222,87 \%)$ handling anti-cancer MABs. 95 (43\%) respondents reported access to institutional MAB handling guidelines. Among those with guidelines, 74 (78\%) reported 'good' compliance to recommendations. Strategies implemented to reduce the risk of occupational are presented in table 2. Among clinicians providing an evaluation of institutional guidelines, the most common strategies employed to reduce risk were training and education (71\%), spill and waste management (71\%), procedures for transportation (57\%) and restricted handling (50\%).

For all MABs included in the survey, the majority of clinicians ( $84 \%$ to $90 \%$ ) prepared (manufactured) agents onsite. For the range of MABs included in the survey, the majority of respondents (overall median: $75 \%$, overall range: $37 \%$ to $88 \%$ ) prepared MABs in pharmacy clean-rooms, figure $1.61 \%$ utilised cytotoxic down flow cabinets, $15 \%$ closed system drug transfer devices (CSDTD), $6.8 \%$ laminar cross flow cabinets, $4.8 \%$ 
biohazard cabinets and $2.9 \%$ clean area within pharmacy. Variation in reported handling practices was most notable for denosumab and trastuzumab. Fifty one percent of clinician's reported preparation of denosumab on the ward compared with $38 \%$ in a pharmacy cleanroom, with an opposing trend for trastuzumab; $11 \%$ prepared on the ward compared with $74 \%$ in a pharmacy clean room. Reported use of personal protective equipment (PPE) varied among agents and among respondents, figure 2.

Attitudes regarding the occupational exposure risk and hence required handling procedures for MABs are reported for 172 clinicians, with notable differences by profession. $80 \%$ of nurses compared to $50 \%$ of doctors $(p=0.011)$ and $44 \%(0.001)$ of pharmacists, strongly agreed that all MAB admixtures should be prepared in pharmacy clean rooms. $70 \%$ of nurses compared with $51 \%$ of doctors $(p=0.014)$ and $45 \%$ of pharmacists $(p=0.015)$ strongly agreed that drugs to be given to immunocompromised patients should be prepared in pharmacy clean rooms. $80 \%$ of nurses compared with $49 \%$ of doctors $(p=0.008)$ and $65 \%$ of pharmacists $(p=0.129)$ strongly agreed that drugs with complex manufacturing requirements should be prepared in pharmacy clean rooms. Nurses had a stronger preference toward pharmacy manufacturing than both doctors and pharmacists for a range of additional clinical scenarios illustrated in figure $\mathbf{3}$.

When asked on the importance of independently produced guidelines (external regulatory or professional body guidelines) for the handling of MABs, of those who responded, 21/28 (75\%) nurses, 62/95 (65\%) pharmacists and 15/35 (43\%) doctors strongly agreed that they are important in considering handling practices. Factors influencing MAB handling practices, presented by respondent profession, are described in

figure 4. All groups, (170/172, 99\% of respondents), agreed that evidence of mutagenicity, teratogenicity or carcinogenesis in patients of healthcare workers is an important factor when considering the location for the preparation of MABs. The molecular size and weight of MABs (magnitudes higher than traditional cytotoxic chemotherapy), was considered important (agree or strongly agree) by $31 \%, 45 \%$ and $54 \%$ of medical, pharmacy and nursing personnel respectively. Other factors reported to influence preparation included pharmaceutical company and professional or regulatory body guidance, the type of preparation (e.g. formulation, number of vials required and complexity of manipulations) as well as the ability to provide timely access to treatment.

\section{Discussion:}

This is the first published study reporting handling practices of MABs in healthcare services across Australia. More than $90 \%$ of respondents reported preparation, handling or administration of MABs at their healthcare institution, yet less than 50\% reported access to MAB handling guidelines. Furthermore, respondents from all professional groups ( $>95 \%$ of all respondents) identified that professional or regulatory body information (i.e. independently produced guidelines) are an important resource when considering handling practices. The results are felt to be generalisable acrossAustralia with responses from a variety (geographic location, size and services offered) of healthcare institutions. Acknowledgment should however be given to the large proportion of Victorian respondents, a potential bias and likely influence from key project personnel who practice in 
Victorian hospitals. Reassuringly, project personnel were dispersed across five Victorian healthcare services ranging from specialist oncology centres to large general hospitals.

Survey results demonstrate that the majority of MABs are prepared using full cytotoxic precautions (cytotoxic cabinet in pharmacy, full personal protective equipment). This common practice remains despite neither NOHSC (Safe Work Australia) nor NIOSH listing these agents as hazardous substances, perhaps demonstrating a belief that this is an omission of classification rather than classification that these agents are truly not hazardous. This is supported by findings that respondents equally preference (ideal manufacturing practices) the pharmacy manufacturing of brentuximab vedotin (MAB conjugated to a cytotoxic agent) and cetuximab (unconjugated $\mathrm{MAB}$ ), $83 \%$ and $80 \%$ of respondents respectively.

Alternatively, this may reflect a practical (rather than occupational health and safety) rationale for manufacturing MABs in a pharmacy clean room. Benefits of manufacturing in a pharmacy clean-room include an ability to vial share (financial savings) and restriction of manufacturing to highly trained staff. Restricting manufacturing may theoretically reduce the risk of drug damage (MABs being sensitive proteinaceous compounds) or manufacturing error (e.g. wrong dose, volume or diluent) associated with incorrect handling and complex manipulations. Restriction of preparation to experienced or well-trained personnel (whether in a pharmacy clean room or not), has been demonstrated to reduce levels of microbial contamination in aseptically prepared parenteral medications, ${ }^{27-29}$ which may provide additional motivation for centralised manufacturing. Survey results indicate that a combination of occupational health and safety and practical considerations were taken into account when deciding where MABs should be prepared.

The majority (99\%) of clinicians believed that evidence of mutagenicity, teratogenicity or carcinogenicity of a drug in patients or healthcare workers was an important factor when considering appropriate handling practices. This belief system aligns with current hazardous substance criteria, ${ }^{1,2}$ however assumes firstly that this information is readily available, and secondly that there is a mechanism for internalisation (systemic bioavailability). Information relating to the toxicological profile of MABs is not as readily available as conventional medicines. As biotechnology derived products, MABs are not subject to the same testing as conventional medicines. According to the International Conference on Harmonisation of Technical Requirements for Registration of Pharmaceuticals for Human Use (ICH) guidelines, as pharmaceutical products derived from biotechnology there is no requirement for MABs to be evaluated for either carcinogenicity or genotoxicity. ${ }^{30}$ Although not formally tested for genotoxicity, following administration, MABs are limited to the extracellular space and do not directly interact with DNA (hence are not directly genotoxic). This point of view is agreed on by the American College of Toxicology (ACT) and German Society of Toxicology who state that there is little to no concern that bio-therapeutics may induce a genotoxic insult. ${ }^{31}$ Furthermore, differing from traditional anticancer agents, MABs do not exhibit direct cytotoxic activity. They do however function as 'cytotoxic agents' with immune-mediated cytotoxicity, or more specifically antibody-dependent cellular cytotoxicity $(A D C C)$, being a major mechanism of action. ${ }^{32,33}$ The relevance of this indirect cytotoxic activity in regard to occupational exposure is unknown, however as with other systemic effects, internalisation is the rate 
limiting step. The ability for MABs to be internalised by healthcare workers during routine occupational operations is unclear. NIOSH, to date have justified exclusion of MABs from their hazardous substance register based on the belief that internalisation is improbable. ${ }^{22}$ Accordingly, the common current practice of preparing MABs using full cytotoxic precautions is not supported by major occupational health and safety organisations. ${ }^{1}$ 2 A full review of available evidence, including pharmacologic principles and mechanistic internalisation evaluations for MABs, within the occupational setting, is required.

Comparison of clinician attitudes regarding the occupational exposure risks and hence required handling precautions for MABs, demonstrated an overall trend (often reaching statistical significance) that nurses were more conservative and seeking greater guidance than pharmacists, who in turn were more conservative than medical staff. Practically, this may reflect the work-load of 'on the floor' nursing staff, whom on the basis of time allocation alone may prefer not to manufacture / prepare agents. Differing attitudes among the professional groups are reflective of the level of potential exposure risk associated with each profession. Nursing staff are involved in both handling and administration (and sometimes preparation), pharmacy staff only in the preparation and medical staff either not at all, or in the administration process only. This may also reflect differing knowledge and understandings of potential occupational exposure risks associated with MABs, highlighting that important issue of staff training and education relating to occupational health and safety risks. Results suggestive of a knowledge gap across all professions are that just $77 / 131$ (45\%) of all respondents ( $53 \%$ of nurses, $45 \%$ of pharmacists, $31 \%$ of doctors), considered the molecular size of MABs to be an important factor when considering exposure risk. The large molecular size of MABs, typically greater than 140 kilo Daltons, is orders of magnitude greater than traditional anticancer agents; molecular weight of gemcitabine 299.66 Daltons. ${ }^{34}$ More importantly, it is orders of magnitude greater than agents used for topical or transdermal drug delivery and of agents which are known contact allergens, which are typically less than 500 Daltons. ${ }^{35}$ This strongly indicates that there is no pharmacologic mechanism for the dermal absorption of MABs.

$46 \%$ of pharmacists compared with $61 \%$ of nurses strongly agreed that the complexity of manipulation and preparation is an important factor to consider in regard to the preparation of MABs. The processes involved in the preparation of these molecules are often complex, with numerous and multifaceted manipulations involved. A number of these drugs also have special instructions which need to be followed during the preparation process. For example, do not shake excessively, direct the stream of the diluent into or away from the powder in order to maintain integrity of the final product. Additionally, the calculations involved in determining the correct volumes of drug and diluent are often complex. The risk of error associated with preparation in a ward environment is not insignificant with studies reporting frequencies of clinical interruptions at up to 5.2 interruptions per hour of nursing medication preparation time. ${ }^{36}$ In a busy ward environment, nursing staff may not have the time to prepare these drugs, particularly if complex processes are involved. Additionally, these agents may be used infrequently or use spread across multiple clinical areas of an individual hospital or institution and consequently ward staff may not be familiar with the processes involved. 
The Australian Federal Government pays a compounding fee of $\$ 40$ for the specialist requirements of preparing 'chemotherapy' medicine (currently bevacizumab, cetuximab, rituximab and trastuzumab receive funding in this manner). ${ }^{37}$ This fee is paid regardless of whether reconstitution/manufacturing occurs in the pharmacy, by staff on the ward or by a third party compounder. Attachment of a compounding fee therefore should not influence decisions regarding preparation of MABs. However survey results indicate that this is an important consideration with $68 \%$ of respondents citing PBS compounding fee payment as a determinant of manufacturing practices. Future research evaluating the economic impact of MAB (and other pharmaceuticals) handling practices would further inform policy in this area.

The results of this survey demonstrate that in the Australian healthcare setting, anticancer MABs are most commonly handled according to cytotoxic drug standards, and most commonly in the absence of formal guidelines. Attitudes and beliefs of the occupational health and safety risk associated with these agents vary by profession and are divergent from the views of national and international occupational health and safety authorities. Respondents identified a need for guidelines for the handling of MABs.Concordantly, results from this survey were used to inform the development of Australian consensus guidelines for the safe handling of monoclonal antibodies for cancer treatment by healthcare personnel (available via the Western and Central Melbourne Integrated Cancer Services (WCMICS) website - http://www.wcmics.org/). Informed by the survey finding that both occupational and non-occupational health and safety issues were important factors to consider when determining how and where a MAB should be prepared, the developed guidelines recognise both considerations. Within the guidelines, in addition to definitive recommendations for the minimum safe handling requirements to protect healthcare personnel, a risk assessment model is also includedto allow institutions to consider and evaluate clinical and operational (non-occupational health and safety) site specific factors.

\section{Acknowledgements}

This project was funded by Western and Central Melbourne Integrated Cancer Service (WCMICS) as part of a project commissioned to develop safe handling guidelines for MABs. 


\section{Figure Legend}

Figure 1 - Current versus Ideal MAB manufacturing practices

Figure 2 - Use of Personal Protective Equipment (PPE) during MAB preparation

Figure 3 - Attitudes regarding the handling of MABs by profession

Figure 4 - Factors influencing decisions regarding handling precautions and manufacturing location by profession 
1. List of antineoplastic and other hazardous drugs in health care settings 2012. National Institute for Occupational Safety and Health (NIOSH); 2012.

2. Approved Criteria for Classifying Hazardous Substances [NOHSC:1008(2004)]. Australian National Standard. 3rd Revision. National Occupational Health and Safety Commission (NOHSC); 2004.

3. EC Council Directive 67/548/EEC on the approximation of the laws raaprttc, packaging and labelling of dangerous substances, Official Journal of the European Communities, No. L196 (16 August 1967).

4. Labelling of workplace hazardous chemicals. Code of Practice [section 274 of the Work Health and Safety Act]. December 2011. Safe Work Australia.

5. Adams J and Kauffman M. Development of the Proteasome Inhibitor Velcade ${ }^{\mathrm{TM}}$ (Bortezomib). Cancer Investigation. 2004; 22: 304-11.

6. ASHP technical assistance bulletin on handling cytotoxic and hazardous drugs. American Journal of Health-System Pharmacy. 1990; 47: 1033-49.

7. Victorian Government. Occupational Health and Safety Regulations. S.R. No. 54/2007. Version No. 001: (2007).

8. NIOSH. NIOSH Alert Preventing Occupational Exposures to Antineoplastic and Other Hazardous Drugs in Health Care. Publication no 2004-165National Institute for Occupational Safety and Health: (2004).

9. Dangerous Goods Act 1985. Act No. 10189/1985. Version No. 081. (1985). Retrieved from www.austlii.edu.au: (1985).

10. Victorian Government. Dangerous Goods (Storage and Handling) Regulations. S.R. No. 132/2012: (2012).

11. SHPA. Standards of practice for the safe handling of cytotoxic drugs in pharmacy departments. Journal of Pharmacy Practice and Research. 2004; 35: 44-52.

12. WorkSafe Victoria. Handling cytotoxic crugs in the workplace. Available from http://www.worksafe.vic.gov.au/_data/assets/pdf_file/0010/12223/handling_cytotoxic.pdf.pdf: (2003).

13. Safe handling of hazardous drugs and related wastes: Guidelines for South Australian Health Services. Version 1.0. Department of Health, Government of South Australia: (2012).

14. SHPA. Guidelines for medicines prepared in australian hospital pharmacy departments. Journal of Pharmacy Practice and Research. 2010; 40: 133-43.

15. Ginn SL, Alexander IE, Edelstein ML, Abedi MR and Wixon J. Gene therapy clinical trials worldwide to 2012 - an update. The Journal of Gene Medicine. 2013; 15: 65-77.

16. Bortezomib (Velcade) Product Information. Jannsen. Revision date: 20/11/2012.

17. Connor T, McLauchlan R and Vandenbroucke J. ISOPP Standards of Practice - Cytotoxics. J Oncol Pharm Pract. 2007; Supplement to 13: 13-81.

18. The Scientific Advisory Committee on Genetic Modification (SACGM) compendium of guidance. Part 6: guidance on the use of genetically modified microorganisms in a clinical setting. Health and Safety Executive (HSE). First published 01/07.

19. Clinical Oncology Society of Australia, Cancer Pharmacists Group Position Statement. Safe handling of monoclonal antibodies in healthcare settings. Melbourne: Clinical Oncology Society of Australia; 2013.

20. Kaestli L, Fonzo-Christe C, Bonfillon C, Desmeules J and Bonnabry P. Development of a standardised method to recommend protective measures to handle hazardous drugs in hospitals. Eur J Hosp Pharm. 2013; 20: 100-5.

21. Halsen $\mathrm{G}$ and Kramer I. Assessing the risk to health care staff from long-term exposure to anticancer drugs--the case of monoclonal antibodies. J Oncol Pharm Pract. 2011; 17: 68-80.

22. Langford S, Fradgley S, Evans $\mathrm{M}$ and Blanks $\mathrm{C}$. Assessing the risk of handling monoclonal antibodies. Hospital Pharmacist. 2008; 15: 60-4. 
23. Guidance on the safe handling of monoclonal antibody (MAB) products. 4th Edition. National Health Service (NHS) Pharmaceutical Quality Assurance Committee; 2008

24. Guideline for the preparation or manipulation of monoclonal antibodies (MABs) and related compounds such as fusion proteins, used in the treatment of cancer. Version 2.0. Pan Birmingham Cancer Network; 2012.

25. Monoclonal antibodies risk management for Northern Sydney Local Health District (NSLHD). Document number PR2013_001. Department of Oncology, Division of Medicine, Northern Sydney Local Health District hospitals; 2013.

26. Pigneret-Bernard S, Saint-Lorant $G$ and Divanon F. Safety of monoclonal antibodies for the technician: myth or reality? About a survey of professional practice. J Pharm Clin. 2008; 27.

27. Austin $\mathrm{P}$ and Elia M. Improved aseptic technique can reduce variable contamination rates of ward-prepared parenteral doses. Journal of Hospital Infection. 2013; 83: 160-3.

28. Thomas M, Sanborn M and Couldry R. I.V. admixture contamination rates: Traditional practice site versus a class 1000 cleanroom. Am J Health Syst Pharm. 2005; 62: 2386-92.

29. Trissel L, Gentempo J, Anderson R and LaJeunesse J. Using a medium-fill simulation to evaluate the microbial contamination rate for USP medium-risk-level compounding. Am J Health Syst Pharm. 2005; 62: 285-8.

30. Guidance for Industry S6 Preclinical Safety Evaluation of Biotechnology-Derived Pharmaceuticals (ICH S6). International Conference on Harmonisation of Technical Requirements for Registration of Pharmaceuticals for Human Use (ICH); 1997.

31. Kerwin BA. Polysorbates 20 and 80 used in the formulation of protein biotherapeutics: structure and degradation pathways. Journal of pharmaceutical sciences. 2008; 97: 2924-35.

32. Mellor JD, Brown MP, Irving HR, Zalcberg JR and Dobrovic A. A critical review of the role of Fc gamma receptor polymorphisms in the response to monoclonal antibodies in cancer. Journal of Hematology \& Oncology. 2013; 6: 1-10.

33. Overdijk MB, Verploegen $\mathrm{S}$, van den Brakel JH, et al. Epidermal growth factor receptor (EGFR) antibody-induced antibody-dependent cellular cytotoxicity plays a prominent role in inhibiting tumorigenesis, even of tumor cells insensitive to EGFR signaling inhibition. Journal of immunology (Baltimore, Md : 1950). 2011; 187: 3383-90.

34. Lilly E. Gemcitabine (Gemzar). Approved Product Information. ARTG approval date: 02/08/1995. Revision date: 16/06/2013: (2013).

35. Bos JD and Meinardi MM. The 500 Dalton rule for the skin penetration of chemical compounds and drugs. Experimental dermatology. 2000; 9: 165-9.

36. Biron $A D$, Lavoie-Tremblay $M$ and Loiselle $C G$. Characteristics of work interruptions during medication administration. J Nurs Scholarsh. 2009; 41: 330-6.

37. Moss GP, Dearden JC, Patel $\mathrm{H}$ and Cronin MTD. Quantitative structure-permeability relationships (QSPRs) for percutaneous absorption. Toxicology in Vitro. 2002; 16: 299-317. 
Table 1 - Respondent Demographics

\begin{tabular}{|c|c|}
\hline Primary Place of Practice & n (\%) \\
\hline \multicolumn{2}{|l|}{ Location } \\
\hline Victoria & $117(52.7 \%)$ \\
\hline New South Wales & 31 (14\%) \\
\hline Queensland & $24(10.8 \%)$ \\
\hline Western Australia & $12(5.4 \%)$ \\
\hline Other & $22(9.9 \%)$ \\
\hline Not specified & $16(7.2 \%)$ \\
\hline \multicolumn{2}{|l|}{ Rurality } \\
\hline Metropolitan/Major City & 155 (69.8\%) \\
\hline Regional/Rural & $51(23 \%)$ \\
\hline Not specified & $16(7.2 \%)$ \\
\hline \multicolumn{2}{|l|}{ Institution Type } \\
\hline Hospital or Clinic & $196(88.2 \%)$ \\
\hline External pharmacy compounder & $10(4.5 \%)$ \\
\hline Not specified & $16(7.2 \%)$ \\
\hline \multicolumn{2}{|l|}{ Public or Private } \\
\hline Public only & $167(75.2 \%)$ \\
\hline Private only & $32(14.4 \%)$ \\
\hline Public/Private even split & $7(3.2 \%)$ \\
\hline Not specified & $16(7.2 \%)$ \\
\hline \multicolumn{2}{|l|}{ Institution Size } \\
\hline No inpatient beds & $6(2.7 \%)$ \\
\hline$\leq 150$ beds & 55 (24.8\%) \\
\hline 151 to 300 beds & $32(14.4 \%)$ \\
\hline 301 to 500 beds & $52(23.4 \%)$ \\
\hline$\geq 500$ beds & 35 (15.8\%) \\
\hline Not specified & $42(18.9 \%)$ \\
\hline Profession \& Specialisation & n (\%) \\
\hline Doctor & $46(21 \%)$ \\
\hline Medical Oncology & 25 (54.3\%) \\
\hline Haematology & $14(30.4 \%)$ \\
\hline Both & $7(15.2 \%)$ \\
\hline Head of department or tumour stream & $14(30.4 \%)$ \\
\hline Consultant & $29(63.0 \%)$ \\
\hline Fellow or Registrar & $3(6.5 \%)$ \\
\hline Pharmacy Personnel & $113(51 \%)$ \\
\hline Pharmacist & 109 (96.5\%) \\
\hline Pharmacy Technician & $4(3.5 \%)$ \\
\hline Oncology & $72(63.7 \%)$ \\
\hline Non- Oncology & 37 (33.9\%) \\
\hline Clinical & $59(54.1 \%)$ \\
\hline Manufacturing & $30(27.5 \%)$ \\
\hline Management & $24(22.0 \%)$ \\
\hline Nurse & $50(22 \%)$ \\
\hline Oncology & $48(96.0 \%)$ \\
\hline Non- Oncology & $2(4.0 \%)$ \\
\hline Clinical & $38(76.0 \%)$ \\
\hline Management & $4(8.0 \%)$ \\
\hline Other (education, trials, research) & $5(10.0 \%)$ \\
\hline Profession not specified & $14(6 \%)$ \\
\hline
\end{tabular}


Table 2 - Strategies currently employed to reduce employee exposure risk to MABs, $\mathbf{n}(\%)$

\begin{tabular}{l|c}
\hline Strategy & $\mathbf{n}(\%)$ \\
\hline Staff training and education on the potential hazards & $136(70.8 \%)$ \\
\hline Procedures for dealing with spillages and disposal & $136(70.8 \%)$ \\
\hline Procedures for transportation & $109(56.8 \%)$ \\
\hline Restrictions for employees who are pregnant, planning a pregnancy & $95(49.5 \%)$ \\
\hline Storage in a designated, well sign posted section of the work area & $72(37.5 \%)$ \\
\hline Stored separately from other cytotoxic agents & $28(14.6 \%)$ \\
\hline No strategies; no perceived risk to employees handling these agents & $7(3.7 \%)$ \\
\hline No strategies; there are perceived risks, but no action taken & $15(7.8 \%)$ \\
\hline Not Sure & $27(14.1 \%)$ \\
\hline Other & $22(11.5 \%)$ \\
\hline Total Respondents & $192(100.0 \%)$ \\
\hline
\end{tabular}


Figure 1 - Current versus Ideal MAB manufacturing practices

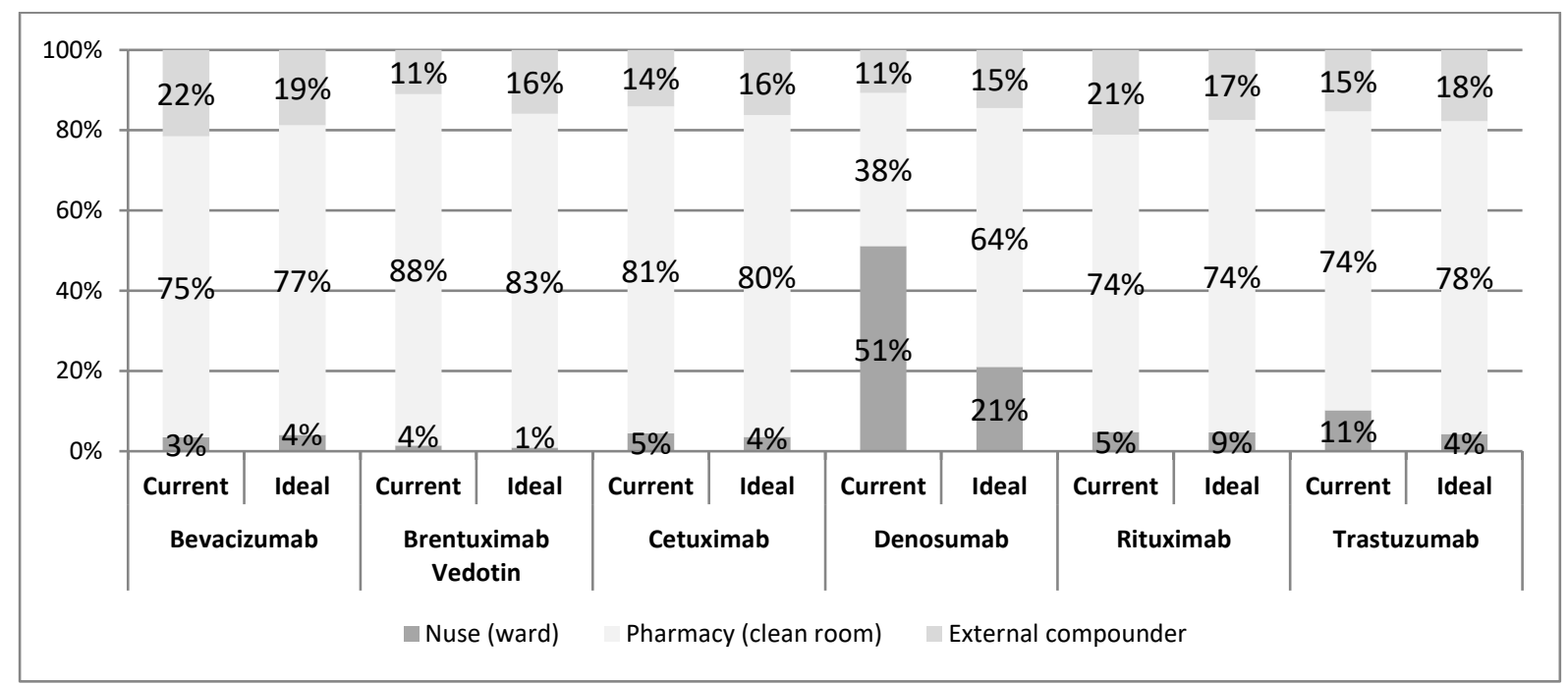

Current - manufacturing practices according to institutional policy; Ideal - manufacturing practices deemed appropriate by survey respondent.

Figure 2 - Use of Personal Protective Equipment (PPE) during MAB preparation

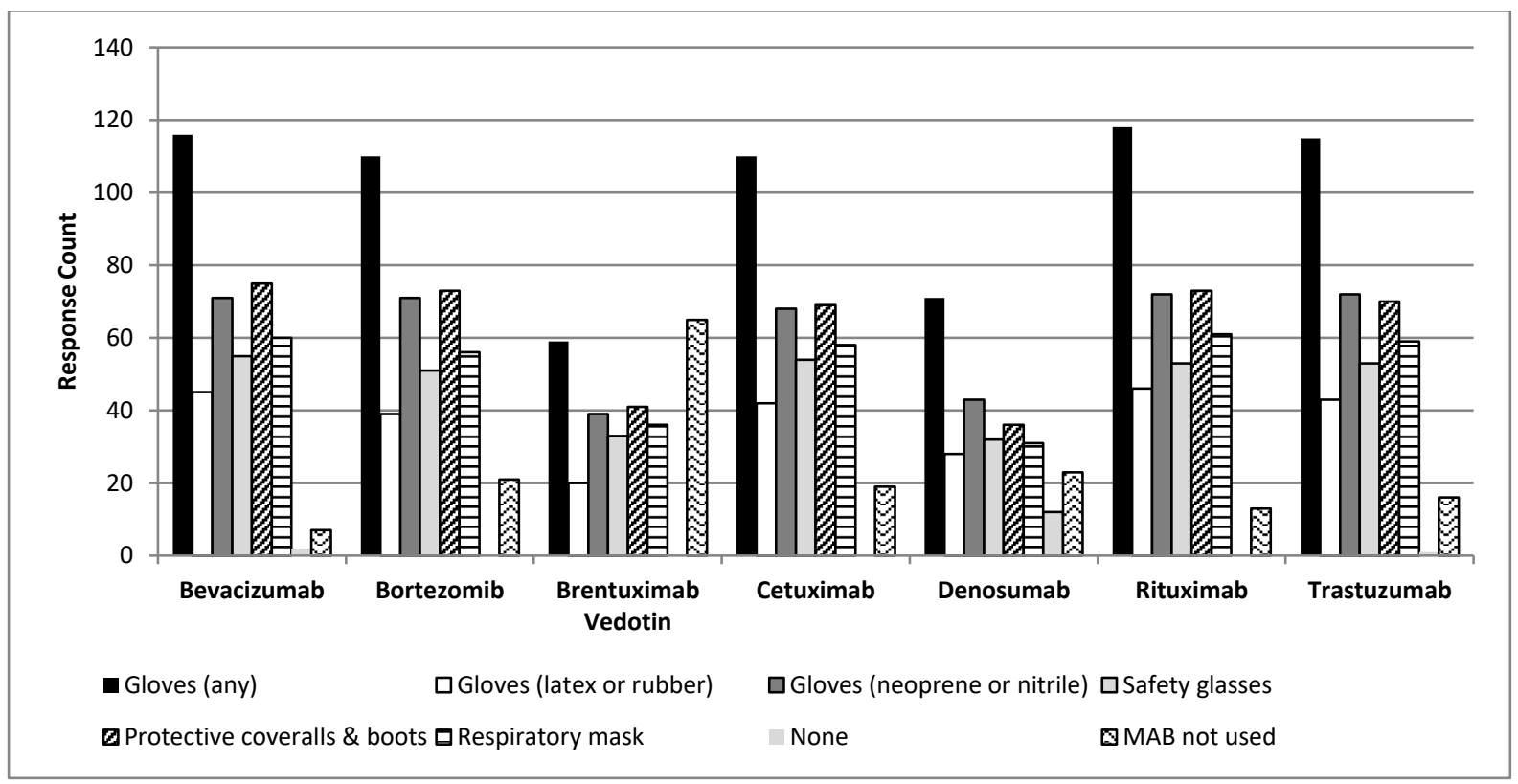


Figure 3 - Attitudes regarding the handling of MABs by profession

Survey Question: Do you agree that MABs should be prepared in a pharmacy clean room?

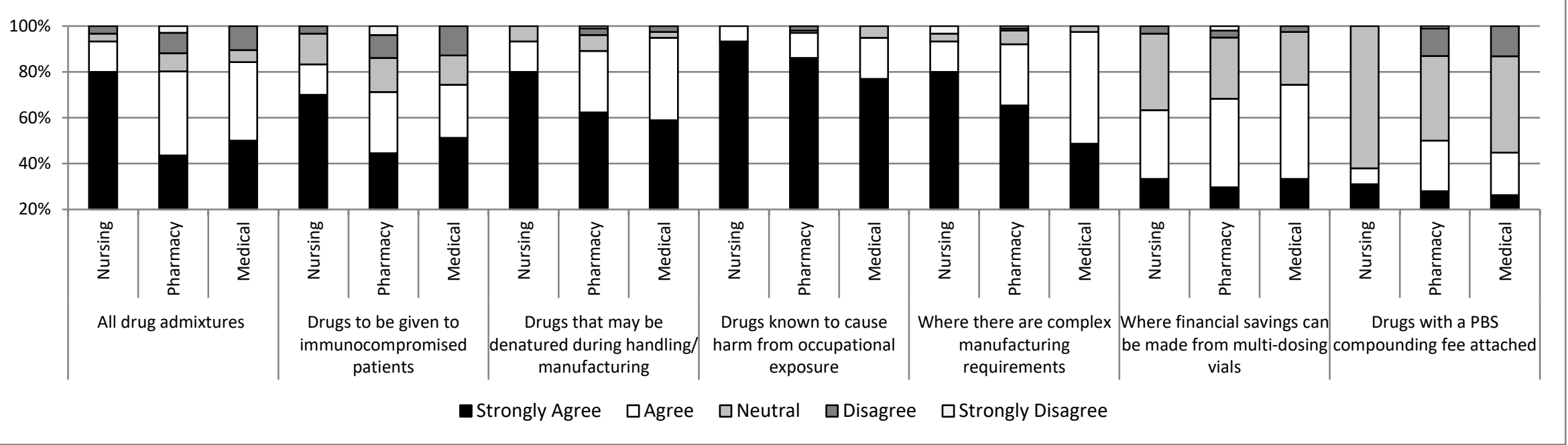


Figure 4 - Factors influencing decisions regarding handling precautions and manufacturing location by profession

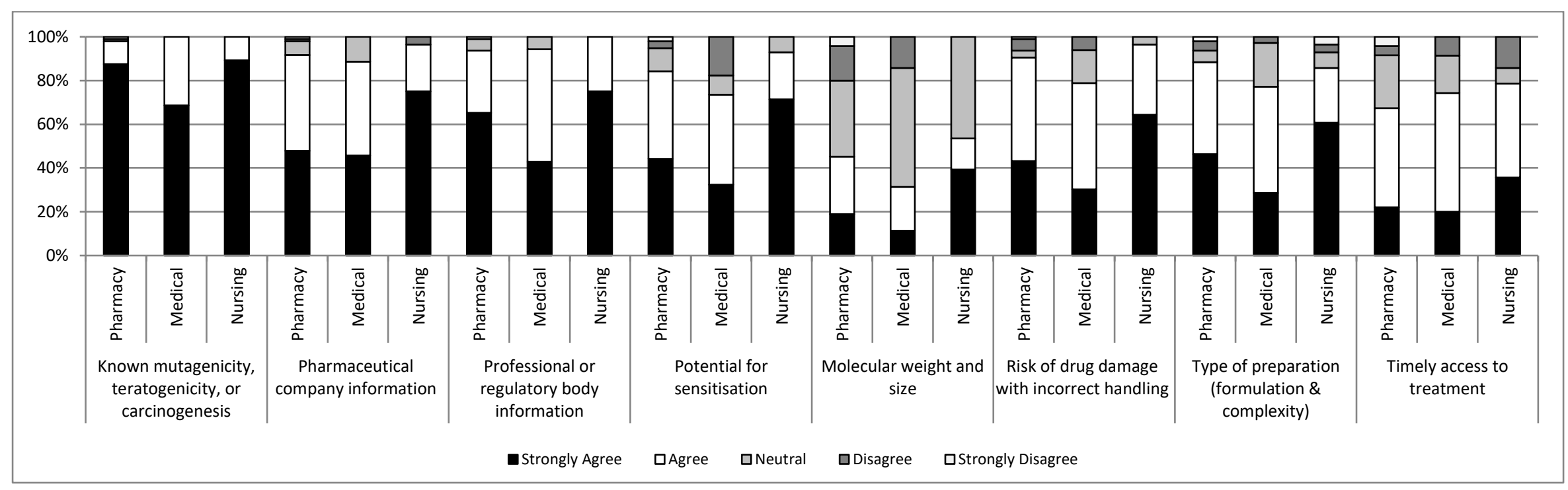

